\title{
A MACROSCOPIC TRAFFIC FLOW MODEL ACCOUNTING FOR BOUNDED ACCELERATION
}

\author{
NICOLAS LAURENT-BROUTY*†, GUILLAUME COSTESEQUE*‡, AND PAOLA GOATIN*
}

\begin{abstract}
This paper details a new macroscopic traffic flow model accounting for the boundedness of traffic acceleration, which is required for physical realism. Our approach relies on the coupling between a scalar conservation law, which refers to the seminal LWR model, and a system of Ordinary Differential Equations describing the trajectories of accelerating vehicles, which we treat as moving constraints. We propose a Wave-Front Tracking Algorithm to construct approximate solutions. We use this algorithm to prove the existence of entropy weak solutions to the associated Cauchy Problem, and provide some numerical simulations illustrating the solution behaviour.
\end{abstract}

Key words. Hyperbolic conservation laws; flux constraint; PDE-ODE systems; wave-front tracking; macroscopic traffic flow models; moving bottlenecks.

AMS subject classifications. 35L65; 90B20.

1. Introduction. Modeling traffic flow using conservation laws started in the mid-fifties with the pioneering works of Lighthill and Whitham [28] and Richards [30]. Assuming conservation of cars on a road, they proposed the so-called Lighthill-Whitham-Richards (LWR) model, which consists in a scalar conservation law in one space dimension

$$
\partial_{t} \rho+\partial_{x} f(\rho)=0, \quad x \in \mathbb{R}, t>0
$$

where $\rho=\rho(t, x) \in\left[0, \rho_{\max }\right]$ denotes the density of cars, $f: \rho \mapsto f(\rho)=\rho v(\rho)$ is the flow-density fundamental diagram and $v: \rho \mapsto v(\rho)$ is a decreasing function representing the mean velocity of the flow. This first model has been afterwards improved, in order to capture additional characteristics of traffic dynamics. We refer the reader to [16] for a recent review of available models. Nonetheless, all these models are based on non-linear conservation laws, and may thus display discontinuous solutions in space and time, giving rise to infinite acceleration or deceleration rates for traffic. In this work, we focus on situations in which the solutions to the LWR model present an unbounded acceleration of traffic. This is the case when the traffic conditions display downward jumps in density, as it happens for example when a traffic light turns green. At these locations, the solution to the classical LWR model consists of a rarefaction wave, accounting for an instantaneous jump from a lower velocity to a higher velocity, which corresponds to an infinite acceleration of the leading vehicle and bounded but unrealistic acceleration values for the following ones. This prevents any coupling of the LWR model (and also second order models like [29, 33] and [3, 34]) with consumption and pollution models (see for example [31] and references therein), in which the acceleration component plays a key role.

Macroscopic models accounting for bounded acceleration of vehicles have been previously addressed in the engineering literature. In particular, [21, 22] propose a two phase model in which the bounded acceleration phase is described by a non-strictly hyperbolic system of balance laws, while [24] applies to rather restrictive cases limited to piecewise affine fundamental diagrams. We propose to couple the LWR model with a finite number of Ordinary Differential Equations (ODEs), each accounting for the trajectory of a leading vehicle, initially located at a downward jump in density, and accelerating at a constant rate. Hence these vehicles act as moving constraints, enforcing a zero flux along their trajectories, until they catch the downstream traffic. In this paper, following our previous work [20], we investigate the well-posedness of our new approach to account for the finite acceleration of vehicles from a macroscopic point of view. We provide a rigorous constructive algorithm to compute approximate solutions to our model, and use it to illustrate the solutions behaviour. Unlike [21, 22, 24, 25], our approach is general and can be extended to a wide class of fundamental diagrams, or even to higher order models.

\footnotetext{
*Inria Sophia Antipolis - Méditerranée, Université Côte d'Azur, Inria, CNRS, LJAD, 2004 route des Lucioles - BP 93,06902 Sophia Antipolis Cedex, France. E-mail: \{nicolas.laurent-brouty, paola.goatin\}@inria.fr

${ }^{\dagger}$ Ecole des Ponts ParisTech, Champs-sur-Marne, France

¥current address: Cerema Ouest, 9 rue René Viviani - BP 46223, 44262 Nantes Cedex 2, France. E-mail: guillaume. costeseque@cerema.fr
} 
The idea of modeling the interaction of specific vehicles (like buses or trucks) with the surrounding traffic through strongly coupled PDE-ODE systems was introduced in [23] and then studied analytically in $[11,15,19,27]$. The interested reader is also referred to $[4,7,12,32]$ for numerical treatments and extensions to second order models. Compared to the above literature, in our paper the moving constraint follows a non-linear trajectory imposed by the acceleration bound, which is independent from the downstream traffic conditions as long as the leading vehicle (i.e. the bottleneck) has not rejoined the preceding vehicles. The bottleneck dynamics, and its impact on traffic, are therefore different from the framework described in [11]. Yet, at this stage, we are only interested in constructing a fine approximation algorithm and in proving existence of solutions for piecewise constant initial data.

The remaining part of the paper is organized as follows: in Section 2 we describe the PDE-ODE model and state the main result. In Section 3, we describe the Wave-Front Tracking algorithm we employ to construct approximate solutions to our model. We then prove that these approximations converge to a entropy weak solution of (2.1) in Section 4. Section 5 is devoted to numerical simulations illustrating a realistic application.

2. A PDE-ODE model for bounded acceleration. Since situations with unbounded acceleration can only appear at downward jumps of density, we propose to couple the LWR model with a moving bottleneck consisting in a single vehicle accelerating at constant rate $A>0$ and originating at the jump location, following the idea of $[11,23]$. In order to transmit its behavior to upstream traffic, we prevent overtaking by adding a flux constraint along the trajectory of each moving bottleneck. To this end, we extend the model introduced in [20], introducing $I$ moving bottlenecks, where $I \in \mathbb{N}$ is the finite number of downward jumps in density at the initial time $t=0$. In the following, we will use the set of indices

$$
\mathcal{I}:=\{1 \leq i \leq I, \quad i \in \mathbb{N}\} .
$$

The model reads as follows:

$$
\begin{aligned}
& \partial_{t} \rho+\partial_{x} f(\rho)=0, \\
& \rho(0, x)=\rho^{0}(x), \\
& f\left(\rho\left(t, y_{i}(t)\right)\right)-\rho\left(t, y_{i}(t)\right) \dot{y}_{i}(t) \leq 0, \\
& \dot{y}_{i}(t)=\omega_{i}\left(t, y_{i}(t)\right) \\
& y_{i}(0)=y_{i}^{0}
\end{aligned}
$$

$$
\begin{aligned}
x \in \mathbb{R}, t & >0, \\
x & \in \mathbb{R}, \\
t>0, & i \in \mathcal{I}, \\
t>0, & i \in \mathcal{I}, \\
i & \in \mathcal{I},
\end{aligned}
$$

where $\rho^{0}$ is a piecewise constant function of $x$, and for every $y_{i}^{0}, i \in \mathcal{I}$, the initial datum has a downward jump: $\rho^{0}\left(y_{i}^{0}-\right)>\rho^{0}\left(y_{i}^{0}+\right)$. In equation (2.1d), we have defined

$$
\omega_{i}\left(t, y_{i}(t)\right):=\min \left\{A t+v_{i}^{0}, v\left(\rho\left(t, y_{i}(t)+\right)\right\}\right.
$$

where $v_{i}^{0}=v\left(\rho^{0}\left(y_{i}^{0}-\right)\right)$ stands for the initial speed of the moving bottleneck starting at position $y_{i}^{0}$ and $A>0$ is the constant acceleration rate assumed to be equal for all vehicles. The map $y_{i}: t \mapsto y_{i}(t)$ denotes the trajectory of the $i$-th moving bottleneck.

In the rest of the paper, we consider the following assumptions:

(A1) The map $v: \rho \mapsto v(\rho)$ is decreasing, Lipschitz continuous and satisfies $v(0)=V_{\max }$ and $v\left(\rho_{\max }\right)=0$.

(A2) The map $f: \rho \mapsto f(\rho)$ is strictly concave, differentiable, satisfies $f(0)=f\left(\rho_{\max }\right)=0$ and we denote $\left.\rho_{\text {cr }} \in\right] 0, \rho_{\max }[$ the point for which $f$ reaches its maximum.

(A3) The initial datum $\rho^{0} \in \mathbf{B V}\left(\mathbb{R} ;\left[0, \rho_{\max }\right]\right)$ is a piecewise constant function with a finite number $I \in \mathbb{N}$ of downward jumps.

Concerning assumption (A3) above, we remark that any function in $\mathbf{B V}\left(\mathbb{R} ;\left[0, \rho_{\max }\right]\right)$ can be approximated by a piecewise constant function with a finite number of jumps, see [5, Lemma 2.2], but we will not treat this general case here. It is noteworthy that in our paper BV spaces are defined following [1, Definition 3.1].

Following $[11,15]$, solutions to (2.1) are intended in the weak sense specified below: 
Definition 1 (Entropy weak solution of the PDE-ODE model). Let $T>0$ be a given finite time horizon. We call $(\rho, y) \in \mathcal{C}^{0}\left([0, T] ; \mathbf{L}^{1}\left(\mathbb{R} ;\left[0, \rho_{\max }\right]\right)\right) \times \mathbf{W}^{1,1}\left([0, T] ; \mathbb{R}^{I}\right)$ a solution to $(2.1)$ if and only if

(i) for all $\kappa \in \mathbb{R}$ and $\phi \in \mathcal{C}_{c}^{1}(]-\infty, T\left[\times \mathbb{R} ; \mathbb{R}^{+}\right)$,

$$
\begin{array}{r}
\int_{0}^{T} \int_{-\infty}^{\infty}\left(|\rho-\kappa| \phi_{t}+\operatorname{sgn}(\rho-\kappa)(f(\rho)-\right. \\
\left.+(\kappa)) \phi_{x}\right) \mathrm{d} x \mathrm{~d} t+\int_{-\infty}^{\infty}\left|\rho^{0}(x)-\kappa\right| \phi(0, x) \mathrm{d} x \\
+2 \sum_{i=1}^{I} \int_{0}^{T}\left(f(\kappa)-\dot{y}_{i}(t) \kappa\right) \phi\left(t, y_{i}(t)\right) \mathrm{d} t \geq 0 ;
\end{array}
$$

(ii) For $i \in \mathcal{I}$, each component $y_{i}$ of $y$ is a Carathéodory solution of (2.1d), (2.1e), i.e.

$$
y_{i}(t)=y_{i}^{0}+\int_{0}^{t} \omega_{i}\left(s, y_{i}(s)\right) \mathrm{d} s \text { for any } t \in[0, T]
$$

(iii) the constraint (2.1c) is satisfied for almost every $t \in[0, T]$ and any $i \in \mathcal{I}$.

Our main result is the following:

Theorem 1 (Existence of a entropy weak solution). Assume that (A1), (A2) and (A3) hold. Then the Cauchy problem (2.1) admits a solution in the sense of Definition 1.

3. Construction of approximate solutions with Wave-Front Tracking. First of all, we need to detail the choice of the Riemann solver we will adopt in this paper.

Definition 2 (The Riemann solver for scalar conservation laws [17]). Assume (A2) holds true. Consider the classical Riemann problem for (1.1) at $x=y^{0} \in \mathbb{R}$

$$
\begin{aligned}
& \partial_{t} \rho+\partial_{x} f(\rho)=0, \quad x \in \mathbb{R}, t>0, \\
& \rho(0, x)=\left\{\begin{array}{lll}
\rho_{L} & \text { if } & x<y^{0}, \\
\rho_{R} & \text { if } & x \geq y^{0} .
\end{array}\right.
\end{aligned}
$$

The unique weak entropy solution to (3.1) is given by

$$
\rho(t, x)=\mathcal{R}\left(\rho_{L}, \rho_{R}\right)(\xi)= \begin{cases}\rho_{L} & \text { if } \rho_{L}<\rho_{R}, \xi<\frac{f\left(\rho_{L}\right)-f\left(\rho_{R}\right)}{\rho_{L}-\rho_{R}} \\ & \text { or } \rho_{L} \geq \rho_{R}, \xi<f^{\prime}\left(\rho_{L}\right), \\ \rho_{R} & \text { if } \rho_{L}<\rho_{R}, \xi>\frac{f\left(\rho_{L}\right)-f\left(\rho_{R}\right)}{\rho_{L}-\rho_{R}} \\ & \text { or } \rho_{L} \geq \rho_{R}, \xi>f^{\prime}\left(\rho_{R}\right), \\ \left(f^{\prime}\right)^{-1}(\xi) & \text { if } \rho_{L} \geq \rho_{R}, f^{\prime}\left(\rho_{L}\right)<\xi<f^{\prime}\left(\rho_{R}\right) .\end{cases}
$$

with $\xi:=\left(x-y^{0}\right) / t$.

Definition 3 (The constrained Riemann solver [11]). Assume (A1) and (A2) hold true. For any $V \in\left[0, V_{\max }\right]$, let $\left.\left.\hat{\rho} \in\right] 0, \rho_{\max }\right]$ such that $v(\hat{\rho})=V$. Consider the constrained Riemann solver proposed in [11], where the scalar conservation law is coupled to a flux constraint with constant maximal velocity $V$ :

$$
\begin{aligned}
& \partial_{t} \rho+\partial_{x} f(\rho)=0, \quad x \in \mathbb{R}, t>0, \\
& \rho(0, x)=\left\{\begin{array}{lll}
\rho_{L} & \text { if } \quad x<y^{0}, \\
\rho_{R} & \text { if } \quad x \geq y^{0},
\end{array}\right. \\
& f(\rho(t, y(t))-\rho(t, y(t)) \dot{y}(t) \leq 0, \quad t>0, \\
& \dot{y}(t)=\min \left\{V, v\left(\rho_{R}\right)\right\} \text {, } \\
& y(0)=y^{0} \text {. }
\end{aligned}
$$

Setting $\xi:=\left(x-y^{0}\right) / t$, we define a solution $\rho(t, x)=\mathcal{R}_{V}\left(\rho_{L}, \rho_{R}\right)(\xi)$ to (3.3) as follows: 
- If $f\left(\mathcal{R}\left(\rho_{L}, \rho_{R}\right)(V)\right)-V \mathcal{R}\left(\rho_{L}, \rho_{R}\right)(V)>0$, then the bottleneck is active and

$$
\mathcal{R}_{V}\left(\rho_{L}, \rho_{R}\right)(\xi)=\left\{\begin{array}{ll}
\mathcal{R}\left(\rho_{L}, \hat{\rho}\right)(\xi), & \text { if } \xi<V, \\
\mathcal{R}\left(0, \rho_{R}\right)(\xi), & \text { if } \xi \geq V,
\end{array} \quad \text { and } \quad y(t)=y^{0}+V t .\right.
$$

- If $f\left(\mathcal{R}\left(\rho_{L}, \rho_{R}\right)(V)\right)-V \mathcal{R}\left(\rho_{L}, \rho_{R}\right)(V) \leq 0$, then the bottleneck is inactive and

$$
\mathcal{R}_{V}\left(\rho_{L}, \rho_{R}\right)(\xi)=\mathcal{R}\left(\rho_{L}, \rho_{R}\right)(\xi) \quad \text { for all } \xi \in \mathbb{R} \quad \text { and } \quad y(t)=y^{0}+\min \left\{V, v\left(\rho_{R}\right)\right\} t .
$$

To construct a sequence of approximate solutions $\left\{\rho_{N}, y_{1, N}, \ldots, y_{I, N}\right\}_{N \in \mathbb{N}}$ to (2.1), we adopt the WaveFront Tracking technique (see for instance [18]). For any $N \in \mathbb{N}$, we set a grid of densities consisting of $2^{N}+1$ points as follows

$$
\mathcal{M}_{N}:=2^{-N} \rho_{\max } \mathbb{N} \cap\left[0, \rho_{\max }\right]
$$

and we denote the mesh size by $\epsilon_{N}:=2^{-N} \rho_{\max }$. In particular, for any distinct $\rho_{1}, \rho_{2} \in \mathcal{M}_{N}$, we have $\left|\rho_{1}-\rho_{2}\right| \geq \epsilon_{N}$. Moreover, we denote by $v_{j, N}$ the speed corresponding to density $\rho_{j, N} \in \mathcal{M}_{N}$, i.e. $v_{j, N}:=$ $v\left(\rho_{j, N}\right)$. We take an approximation $\rho_{N}^{0}$ of the initial datum $\rho^{0}$ taking values on the grid, with the same number and location of discontinuities as $\rho^{0}$, such that $\operatorname{TV}\left(\rho_{N}^{0}\right) \leq \operatorname{TV}\left(\rho^{0}\right)$ (existence is guaranteed by $[5$, Section 2]). In the remaining, we will elude the index $N$ for sake of clarity, when it is not necessary for the computations.

Definition 4 (Interaction time between a moving bottleneck and downstream traffic). Consider $\left\{\rho_{N}, y_{1, N}, \ldots, y_{I, N}\right\}_{N \in \mathbb{N}}$ a sequence of approximate solutions to (2.1) obtained via Wave-Front Tracking. For any $i \in \mathcal{I}, N \in \mathbb{N}$, we define the interaction time $t_{i, N}^{i n t}$ as

$$
t_{i, N}^{i n t}:=\inf \left\{t>0: v\left(\rho_{N}\left(t, y_{i, N}(t)-\right)\right) \geq v\left(\rho_{N}\left(t, y_{i, N}(t)+\right)\right)\right\} .
$$

REMARK 1. The interaction time $t_{i, N}^{i n t}$ corresponds to the instant at which the $i$-th moving bottleneck catches up with downstream traffic if existing, else it corresponds to the moment when the bottleneck reaches the maximal speed. By definition, we deduce that $t_{i, N}^{i n t} \leq \frac{V_{\max }}{A}, \forall i \in \mathcal{I}$. Since the sequence $\left\{t_{i, N}^{i n t}\right\}_{N \in \mathbb{N}}$ is uniformly bounded, there exists a subsequence which converges towards some point $t_{i}^{\text {int }} \leq \frac{V_{\max }}{A}$.

For $i \in \mathcal{I}$, the $i$-th moving bottleneck is considered active up to time $t=t_{i, N}^{\text {int }}$. Until then, in the continuous setting (see [20]), a vehicle accelerating at a constant rate $A>0$ would describe a parabola, defined by

$$
y_{i}(t)=\frac{A}{2} t^{2}+v\left(\rho^{0}\left(y_{i}^{0}-\right)\right) t+y_{i}^{0}, \quad \text { for any } \quad t>0 .
$$

In the discrete setting, to construct our Wave-Front Tracking approximations, we will approximate each parabola by a piecewise linear trajectory, such that the initial slope is equal to $v\left(\rho_{N}^{0}\left(y_{i}^{0}-\right)\right)$, and then it increases along the grid, taking values $v\left(\rho_{N}^{0}\left(y_{i}^{0}-\right)-\epsilon_{N}\right), v\left(\rho_{N}^{0}\left(y_{i}^{0}-\right)-2 \epsilon_{N}\right)$ and so on, as long as the moving bottleneck is active. This construction is thus valid on a time horizon $\left[0, t_{i, N}^{\text {int }}[\right.$, which we partition in subintervals $\left[t_{i, N}^{n}, t_{i, N}^{n+1}\right.$ [, where, for any $n$ small enough, $t_{i, N}^{n}$ is defined for each grid-parameter $N$ such that

$$
\left\{\begin{array}{l}
t_{i, N}^{0}=0, \quad t_{i, N}^{n+1}=t_{i, N}^{n}+\Delta t_{i, N}^{n} \\
\Delta t_{i, N}^{n}:=\frac{1}{A}\left[v\left(\rho_{N}^{0}\left(y_{i}^{0}-\right)-(n+1) \epsilon_{N}\right)-v\left(\rho_{N}^{0}\left(y_{i}^{0}-\right)-n \epsilon_{N}\right)\right] .
\end{array}\right.
$$

The interval $\Delta t_{i, N}^{n}$ corresponds to the time necessary to accelerate between two consecutive velocities on the grid $v\left(\rho_{N}^{0}\left(y_{i}^{0}-\right)-n \epsilon_{N}\right)$ and $v\left(\rho_{N}^{0}\left(y_{i}^{0}-\right)-(n+1) \epsilon_{N}\right)$ at a constant acceleration rate $A$.

The approximate trajectory of the active moving bottleneck $y_{i, N}$ is then defined for each grid-parameter $N$ and $t \in\left[0, t_{i, N}^{\text {int }}[\right.$ as

$$
y_{i, N}(t):=y_{i}^{0}+\sum_{j=0}^{n-1} v\left(\rho_{N}^{0}\left(y_{i}^{0}-\right)-j \epsilon_{N}\right) \Delta t_{i, N}^{j}+v\left(\rho_{N}^{0}\left(y_{i}^{0}-\right)-n \epsilon_{N}\right)\left(t-t_{i, N}^{n}\right), \quad \forall t \in\left[t_{i, N}^{n}, t_{i, N}^{n+1}[.\right.
$$


For $t \geq t_{i, N}^{\mathrm{int}}$, i.e. when the bottleneck is no more active, the trajectory is approximated by:

$$
y_{i, N}(t)=y_{i, N}\left(t_{i, N}^{\mathrm{int}}\right)+\int_{t_{i, N}^{\mathrm{int}}}^{t} v\left(\rho_{N}\left(s, y_{i, N}(s)+\right)\right) d s .
$$

REMARK 2. The comparison between the approximate trajectory in the discrete setting and the parabola in the continuous setting provides that $t_{i, N}^{\text {int }} \geq t_{i}^{\text {int }}, \forall i \in \mathcal{I}, \forall N \in \mathbb{N}$.

We now detail the algorithm to construct the Wave-Front Tracking approximations. Let $T>0$ given. We denote by $\rho_{N}$ the approximate solution (see Fig. 3.1), and we distinguish between the following wave types (all moving with speed given by the Rankine-Hugoniot condition):

- rarefaction jumps are jump discontinuities between a left state $\rho_{L}$ and a right state $\rho_{R}$, such that $\rho_{L}=\rho_{R}+\epsilon_{N}$

- classical shocks are jump discontinuities between a left state $\rho_{L}$ and a right state $\rho_{R}$, such that $\rho_{L}<\rho_{R}$ (thus satisfying Lax entropy condition);

- non-classical shocks are jump discontinuities located along the approximate moving bottleneck trajectories $y_{i, N}$ and defined on the time interval $\left[0, t_{i, N}^{i n t}\right]$, such that $\rho_{L}>\rho_{R}=0$.

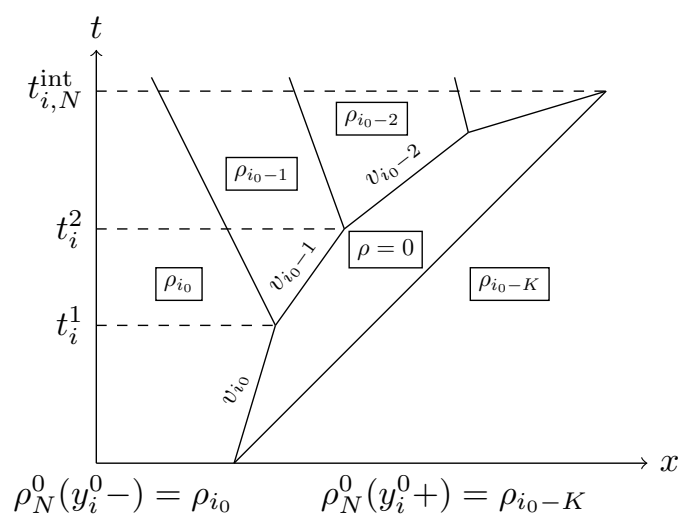

Fig. 3.1: Example of a WFT approximate solution and notation used in the algorithm, focused on the $i$-th bottleneck, where we have set $\rho_{N}^{0}\left(y_{i}^{0}-\right)=: \rho_{i_{0}} \in \mathcal{M}_{N}$ and $v_{j}=v\left(\rho_{j}\right)$.

Algorithm 1 (Wave-Front Tracking (WFT)). Fix $N \in \mathbb{N}$.

Step 0. Approximate the initial datum $x \mapsto \rho^{0}(x)$ by a piecewise constant function $x \mapsto \rho_{N}^{0}(x)$ taking values on the grid $\mathcal{M}_{N}$ defined in (3.4). Denote by $\left\{x_{j}^{0}\right\}_{j=1, \ldots, J}$ the set of $J$ jump locations of $\rho_{N}^{0}$.

Step 1. At $t=0$, using the constrained Riemann solution $\mathcal{R}_{V}$ given by Definition 3 , solve the constrained Riemann problem at every density jump location $x_{j}^{0}$ by setting $V=v\left(\rho_{N}^{0}\left(x_{j}^{0}-\right)\right)$.

- If $\rho^{0}\left(x_{j}^{0}-\right)>\rho^{0}\left(x_{j}^{0}+\right)$ and $x_{j}^{0}=y_{i}^{0}$ for some $i \in \mathcal{I}$, the solution consists in a non-classical shock between $\rho_{N}^{0}\left(y_{i}^{0}-\right)$ and 0 , moving at speed $v\left(\rho_{N}^{0}\left(y_{i}^{0}-\right)\right)$, eventually followed by a classical shock between 0 and $\rho_{N}^{0}\left(y_{i}^{0}+\right)$, moving at speed $v\left(\rho_{N}^{0}\left(y_{i}^{0}+\right)\right)$.

- Else, the solution consists in a classical shock between $\rho_{N}^{0}\left(x_{j}^{0}-\right)$ and $\rho_{N}^{0}\left(x_{j}^{0}+\right)$.

The solution can then be prolonged up to some time $\bar{t}$, the first time at which either an interaction between two waves occurs, or at least one of the approximate moving bottlenecks changes its velocity, for instance bottleneck $y_{i, N}$ at $t_{i}^{1}=\frac{v\left(\rho_{N}^{0}\left(y_{i}^{0}-\right)-\epsilon_{N}\right)-v\left(\rho_{N}^{0}\left(y_{i}^{0}-\right)\right)}{A}$.

Step 2. (i) If $\bar{t}$ is an instant when a change of velocity for an approximate moving bottleneck occurs, $\bar{t}=t_{i}^{1}$ for some $i \in \mathcal{I}$. Then the speed of the approximate moving bottleneck $y_{i, N}$ is set equal to $v\left(\rho_{N}^{0}\left(y_{i}^{0}-\right)-\epsilon_{N}\right)$ and we solve the constrained Riemann problem with $\mathcal{R}_{V}$, where $V=v\left(\rho_{N}^{0}\left(y_{i}^{0}-\right)-\epsilon_{N}\right)$ : the solution consists in a rarefaction jump of size $\epsilon_{N}$ between $\rho_{N}^{0}\left(y_{i}^{0}-\right)$ 
and $\rho_{N}^{0}\left(y_{i}^{0}-\right)-\epsilon_{N}$, moving at speed

$$
\lambda=\frac{f\left(\rho_{N}^{0}\left(y_{i}^{0}-\right)\right)-f\left(\rho_{N}^{0}\left(y_{i}^{0}-\right)-\epsilon_{N}\right)}{\epsilon_{N}},
$$

followed by a non-classical shock between $\rho_{N}^{0}\left(y_{i}^{0}-\right)-\epsilon_{N}$ and 0 , with speed $v\left(\rho_{N}^{0}\left(y_{i}^{0}-\right)-\epsilon_{N}\right)$. In this case the number of waves increases by one.

(ii) Else, $\bar{t}$ is an interaction time between two waves (it is not restrictive to assume that an interaction between three or more waves cannot occur, by possibly changing slightly their velocities). We denote respectively by $\rho_{L}, \rho_{M}, \rho_{R}$ the traffic densities on the left, middle and right of the interacting waves. Different cases may occur:

(a) Both waves are classical shocks. Then $\rho_{L}<\rho_{M}<\rho_{R}$, and the solution at $t=\bar{t}+$ is a classical shock between $\rho_{L}$ and $\rho_{R}$ (the number of waves diminishes).

(b) The first wave is a classical shock and the second wave is a rarefaction jump. Then $\rho_{L}<\rho_{M}=\rho_{R}+\epsilon_{N}$, and thus $\rho_{L}<\rho_{R}$. The solution at $t=\bar{t}+$ is a classical shock between $\rho_{L}$ and $\rho_{R}$ (again, the number of waves diminishes).

(c) The first wave is a rarefaction jump and the second wave is a classical shock. Then $\rho_{L}=\rho_{M}+\epsilon_{N}, \rho_{M}<\rho_{R}$ and $\rho_{L} \leq \rho_{R}$. The solution at $t=\bar{t}+$ is either a classical shock between $\rho_{L}$ and $\rho_{R}$ or no wave is produced if $\rho_{L}=\rho_{R}$ (and the number of waves diminishes).

(d) The first wave is a non-classical shock and the second wave is a classical shock, which yields $\rho_{M}=0$. The concavity of the flux function implies that an interaction occurs if and only if $\rho_{L}<\rho_{R}$. Then the solution at $t=\bar{t}+$ is a classical shock between $\rho_{L}$ and $\rho_{R}$ (and the number of waves diminishes).

Step 3. We repeat Step 2 until $t=T$.

Remark 3. Step 2 (ii) lists all interactions that can possibly occur. Due to the strict concavity of the flux function $f$ (see assumption (A2)), the following cases cannot happen:

- The first wave is a classical shock and the second wave is a nonclassical shock. Then $\rho_{L}<\rho_{M}$ and $\rho_{R}=0$. Hence the first wave moves at speed $\frac{f\left(\rho_{M}\right)-f\left(\rho_{L}\right)}{\rho_{M}-\rho_{L}}$ and the second at speed $\frac{f\left(\rho_{M}\right)}{\rho_{M}}$, but $\frac{f\left(\rho_{M}\right)-f\left(\rho_{L}\right)}{\rho_{M}-\rho_{L}}<\frac{f\left(\rho_{M}\right)}{\rho_{M}}$ by concavity of $f$.

- Both waves are rarefaction jumps: $\rho_{L}>\rho_{M}>\rho_{R}$. By concavity of the flux function, this interaction is also not possible.

- The first wave is a rarefaction jump and the second wave is a non-classical shock. Then we also have $\rho_{L}>\rho_{M}>\rho_{R}=0$ and the interaction is in fact not possible.

- The first wave is a non-classical shock and the second wave is a rarefaction jump. This case cannot happen, since we must have $\rho_{M}=0$.

- Both waves are non-classical shocks. This interaction cannot happen as well since $\rho_{M}=\rho_{R}=0$ and thus the first wave would have a lower speed than the second, evolving at $V_{\max }$.

From the description of Algorithm 1, we deduce that the number of waves increases only when a rarefaction jump is generated at a time where an approximate moving bottleneck updates its speed. Nonetheless, for each grid parameter $N$, each approximate moving bottleneck can only change its speed at most $2^{N}$ times. In addition, the number of approximate moving bottlenecks is bounded by assumption (A3). Therefore the number of waves remains finite and the construction can be carried on up to any positive time $T$.

COROLlary 1. As a direct consequence, the following holds:

- A non-classical shock can only be generated at time $t=0$.

- Two non-classical shocks cannot interact.

- A non-classical shock can only interact with a classical shock. In this case, only a classical shock is generated.

4. Proof of Theorem 1. We divide the proof in various steps. 
4.1. Convergence of approximate solutions. For each solution $\rho_{N}$ constructed via Wave-Front Tracking, we define the following Glimm-type functional:

$$
\Upsilon(t)=\Upsilon\left(\rho_{N}(t, \cdot)\right)= \begin{cases}\operatorname{TV}\left(\rho_{N}^{0}(\cdot)\right)+2 \sum_{i=1}^{I} \rho_{N}^{0}\left(y_{i}^{0}+\right) & \text { if } t=0, \\ \operatorname{TV}\left(\rho_{N}(t, \cdot)\right) & \text { if } t>0 .\end{cases}
$$

LEMMA 1. The map $t \rightarrow \Upsilon(t)$ is non-increasing.

Proof. It is easy to check that $\Upsilon(0) \geq \Upsilon(0+)$. Indeed, the total variation can increase only when a moving bottleneck originates between two states $\rho_{N}^{0}\left(y_{i}^{0}-\right)>\rho_{N}^{0}\left(y_{i}^{0}+\right)$, and an intermediate vacuum state is introduced, increasing the total variation by $2 \rho_{N}^{0}\left(y_{i}^{0}+\right)$. We assume now that two wave fronts interact or a moving bottleneck changes speed at time $\bar{t}>0$. As detailed in Step 2 of Algorithm 1, the total variation never increases in such situations, therefore $\Upsilon\left(\bar{t}^{+}\right) \leq \Upsilon(\bar{t}-)$.

Lemma 2. There exists a subsequence of $\left\{\rho_{N}\right\}_{N \in \mathbb{N}}$ which converges to some function $\rho$ in $\mathbf{L}_{\text {loc }}^{1}$.

Proof. From Lemma 1 and assumption (A3), we deduce that, for any $N \in \mathbb{N}$ and any $t>0$, we have $\operatorname{TV}\left(\rho_{N}(t, \cdot)\right) \leq \operatorname{TV}\left(\rho_{N}^{0}(\cdot)\right)+2 \sum_{i=1}^{I} \rho_{N}^{0}\left(y_{i}^{0}+\right) \leq C$, for some constant $C>0$ independent of $N$. Note that $\left|\rho_{N}(t, x)\right| \leq \rho_{\max }$ for any $(t, x) \in[0, T] \times \mathbb{R}$.

We must prove that for all $s, t \geq 0$, we have:

$$
\int_{-\infty}^{\infty}\left|\rho_{N}(t, x)-\rho_{N}(s, x)\right| \mathrm{d} x \leq L|t-s|
$$

for some $L$ independent of $N$. By construction of the WFT approximations, the speeds of wave fronts in $\rho_{N}(t, \cdot)$ are uniformly bounded by $\left\|f^{\prime}\right\|_{\mathbf{L}^{\infty}\left(\left[0, \rho_{\max }\right]\right)}$, since the approximate solutions take values in $\left[0, \rho_{\max }\right]$. Inequality (4.1) thus holds with $L=C\left\|f^{\prime}\right\|_{\mathbf{L}^{\infty}\left(\left[0, \rho_{\max }\right]\right)}$. We refer the reader to [5, Section 6] for additional details.

We can then apply Helly's theorem [5, Theorem 2.4], which ensures that there exists a subsequence, that we still denote by $\left\{\rho_{N}\right\}_{N \in \mathbb{N}}$, converging to a limit $\rho$ in $\mathbf{L}_{\text {loc }}^{1}([0, \infty[\times \mathbb{R} ; \mathbb{R})$. The limit satisfies

$$
\int_{-\infty}^{\infty}|\rho(t, x)-\rho(s, x)| d x \leq L|t-s| \text { for all } t, s \geq 0 .
$$

Moreover, by almost everywhere pointwise convergence, we have that $\rho(t, x) \in\left[0, \rho_{\max }\right]$ for a.e. $t>0$, $x \in \mathbb{R}$.

We then show the convergence of each piecewise linear bottleneck trajectory $y_{i, N}$ towards the parabola described by the $i$-th accelerating vehicle.

Lemma 3. For any $i \in \mathcal{I}$ and for any $T>0$, there exists a subsequence of $\left\{y_{i, N}\right\}_{N \in \mathbb{N}}$ which converges uniformly to some function $y_{i}$ on $[0, T]$.

Proof. Since the sequence $\left\{y_{i, N}\right\}_{N \in \mathbb{N}}$ is uniformly bounded on any interval $[0, T]$ and equicontinuous, Ascoli-Arzela Theorem guarantees existence of a subsequence uniformly convergent.

Lemma 4. For any $i \in \mathcal{I}$ the limit function $y_{i}$ satisfies:

$$
y_{i}(t)=\frac{A}{2} t^{2}+v\left(\rho^{0}\left(y_{i}^{0}-\right)\right) t+y_{i}^{0} \quad \text { for } t \in\left[0, t_{i}^{i n t}[.\right.
$$

Proof. Let $i \in \mathcal{I}$ and $n \in \mathbb{N}$. We define

$$
\bar{y}_{i}(t)=\frac{A}{2} t^{2}+v\left(\rho^{0}\left(y_{i}^{0}-\right)\right) t+y_{i}^{0} \text { for } t<t_{i}^{\text {int }} .
$$

For any $t \in\left[t_{i}^{n}, t_{i}^{n+1}[\right.$, we have

$$
\dot{\bar{y}}_{i}(t)-\dot{y}_{i, N}(t)=A t+v\left(\rho^{0}\left(y_{i}^{0}-\right)\right)-v\left(\rho_{N}^{0}\left(y_{i}^{0}-\right)-n \epsilon_{N}\right)
$$




$$
\begin{aligned}
& =v\left(\rho^{0}\left(y_{i}^{0}-\right)\right)-v\left(\rho_{N}^{0}\left(y_{i}^{0}-\right)-n \epsilon_{N}\right)+A t_{i}^{n}+A\left(t-t_{i}^{n}\right) \\
& =v\left(\rho^{0}\left(y_{i}^{0}-\right)\right)-v\left(\rho_{N}^{0}\left(y_{i}^{0}-\right)-n \epsilon_{N}\right)+A\left(t-t_{i}^{n}\right) \\
& \quad \quad+A \sum_{l=0}^{n-1} \frac{1}{A}\left[v\left(\rho_{N}^{0}\left(y_{i}^{0}-\right)-(l+1) \epsilon_{N}\right)-v\left(\rho_{N}^{0}\left(y_{i}^{0}-\right)-l \epsilon_{N}\right)\right] \\
& \quad A\left(t-t_{i}^{n}\right) .
\end{aligned}
$$

For any $T \in\left[t_{i}^{n}, t_{i}^{n+1}\left[\right.\right.$, since $y_{i, N}$ is differentiable almost everywhere, we can write:

$$
\begin{aligned}
\bar{y}_{i}(T)-y_{i, N}(T) & =\int_{0}^{T}\left(\dot{\bar{y}}_{i}(t)-\dot{y}_{i, N}(t)\right) \mathrm{d} t \\
& =\sum_{l=0}^{n-1} \int_{t_{i}^{l}}^{t_{i}^{l+1}}\left(\dot{\bar{y}}_{i}(t)-\dot{y}_{i, N}(t)\right) \mathrm{d} t+\int_{t_{i}^{n}}^{T}\left(\dot{\bar{y}}_{i}(t)-\dot{y}_{i, N}(t)\right) \mathrm{d} t \\
& =\frac{A}{2} \sum_{l=0}^{n-1}\left(\Delta t_{i, N}^{l}\right)^{2}+\frac{A}{2}\left(T-t_{i}^{n}\right)^{2} \geq 0
\end{aligned}
$$

We compute

$$
\begin{aligned}
\sum_{l=0}^{n-1}\left(\Delta t_{i, N}^{l}\right)^{2} & =\frac{1}{A^{2}} \sum_{l=0}^{n-1}\left[v\left(\rho_{N}^{0}\left(y_{i}^{0}-\right)-(l+1) \epsilon_{N}\right)-v\left(\rho_{N}^{0}\left(y_{i}^{0}-\right)-l \epsilon_{N}\right)\right]^{2} \\
& \leq \frac{\left\|v^{\prime}\right\|_{\infty}^{2}}{A^{2}} \sum_{i=0}^{n-1} \epsilon_{N}^{2} \\
& =\frac{\left\|v^{\prime}\right\|_{\infty}^{2}}{A^{2}} \sum_{i=0}^{n-1} 2^{-2 N} \underset{N \rightarrow+\infty}{\longrightarrow} 0 .
\end{aligned}
$$

Thus $\left\{y_{i, N}(t)\right\}_{N \in \mathbb{N}}$ converges to $\bar{y}_{i}(t)$ pointwise almost everywhere, and then $y_{i}(t)$ satisfies (4.2).

4.2. Proof of (2.3). We now prove that $\rho:=\lim _{N \rightarrow+\infty} \rho_{N}$ is an entropy weak solution of (2.1a)-(2.1c).

Lemma 5. $\rho$ is an entropy weak solution of (2.1a)-(2.1c), i.e. for all $\kappa \in \mathbb{R}$ and $\phi \in \mathcal{C}_{c}^{1}(]-\infty, T\left[\times \mathbb{R} ; \mathbb{R}^{+}\right)$,

$$
\begin{array}{r}
\int_{0}^{T} \int_{-\infty}^{\infty}\left(|\rho-\kappa| \phi_{t}+\operatorname{sgn}(\rho-\kappa)(f(\rho)-\right. \\
\left.f(\kappa)) \phi_{x}\right) \mathrm{d} x \mathrm{~d} t+\int_{-\infty}^{\infty}\left|\rho^{0}(x)-\kappa\right| \phi(0, x) \mathrm{d} x \\
+2 \sum_{i=1}^{I} \int_{0}^{T}\left(f(\kappa)-\dot{y}_{i}(t) \kappa\right) \phi\left(t, y_{i}(t)\right) \mathrm{d} t \geq 0 .
\end{array}
$$

Proof. All the waves, including the non-classical waves, generated by the Wave-Front Tracking Algorithm 1, satisfy the Rankine-Hugoniot condition. Hence, for any $N$, the function $\rho_{N}$ is a weak entropy solution of the approximated problem

$$
\begin{cases}\partial_{t} \rho+\partial_{x} f_{N}(\rho)=0, & x \in \mathbb{R}, t>0 \\ \rho(0, x)=\rho_{N}^{0}(x), & x \in \mathbb{R}, \\ f_{N}\left(\rho\left(t, y_{i, N}(t)\right)\right)-\rho\left(t, y_{i, N}(t)\right) \dot{y}_{i, N}(t) \leq 0, & t>0, i \in \mathcal{I}\end{cases}
$$

where $f_{N}$ is the piecewise linear function coinciding with $f$ on the grid $\mathcal{M}_{N}$, see [14, Section 3.4]. Therefore, 
$\forall N, \forall \kappa \in \mathbb{R}$ and $\left.\left.\forall \phi \in \mathcal{C}_{c}^{1}(]-\infty, T\right] \times \mathbb{R} ; \mathbb{R}^{+}\right)$, we have

$$
\begin{array}{r}
\int_{0}^{T} \int_{-\infty}^{\infty}\left(\left|\rho_{N}-\kappa\right| \phi_{t}+\operatorname{sgn}\left(\rho_{N}-\kappa\right)\left(f_{N}\left(\rho_{N}\right)-f_{N}(\kappa)\right) \phi_{x}\right) \mathrm{d} x \mathrm{~d} t+\int_{-\infty}^{\infty}\left|\rho_{N}^{0}(x)-\kappa\right| \phi(0, x) \mathrm{d} x \\
+2 \sum_{i=1}^{I} \int_{0}^{T}\left(f_{N}(\kappa)-\dot{y}_{i, N}(t) \kappa\right) \phi\left(t, y_{i, N}(t)\right) \mathrm{d} t \geq 0 ;
\end{array}
$$

Since $f$ is Lipschitz continuous, and since $\rho_{N}$ converges towards $\rho$ in the $\mathbf{L}^{1}$ norm, we can apply the dominated convergence theorem to conclude that $\rho$ is a weak solution of (2.1a), (2.1c) (see also [15]).

4.3. Proof of (2.4). We now prove that $y_{i}:=\lim _{N \rightarrow+\infty} y_{i, N}$ is a Carathéodory solution to (2.1d) and (2.1e), for any $i \in \mathcal{I}$.

We first provide a technical result.

Lemma 6. For any $i \in \mathcal{I}$ and any $N \in \mathbb{N}$, the approximate bottleneck trajectories satisfy

$$
\dot{y}_{i, N}(t)= \begin{cases}v\left(\rho_{N}^{0}\left(y_{i}^{0}-\right)-n \epsilon_{N}\right), & \text { if } t \in\left[t_{i, N}^{n}, t_{i, N}^{n+1}\left[, \leq A t+v\left(\rho_{N}^{0}\left(y_{i}^{0}-\right)\right) .\right.\right. \\ v\left(\rho_{N}\left(t, y_{i, N}(t)+\right)\right), & \text { if } t>t_{i, N}^{i n t},\end{cases}
$$

Proof. We first assume that $t \leq t_{i, N}^{\mathrm{int}}$. Then there exists $n \in \mathbb{N}$ such that $t \in\left[t_{i, N}^{n}, t_{i, N}^{n+1}[\right.$. By construction, we have that

$$
\dot{y}_{i, N}(t)=v\left(\rho_{N}^{0}\left(y_{i}^{0}-\right)-n \epsilon_{N}\right)=A t_{i, N}^{n}+v\left(\rho_{N}^{0}\left(y_{i}^{0}-\right)\right) \leq A t+v\left(\rho_{N}^{0}\left(y_{i}^{0}-\right)\right) .
$$

Now let $t>t_{i, N}^{\text {int }}$. By construction, we have $\dot{y}_{i, N}(t)=v\left(\rho_{N}\left(t, y_{i, N}(t)+\right)\right)$. Let $n \in \mathbb{N}$ such that $t_{i, N}^{\text {int }} \in$ $\left[t_{i, N}^{n}, t_{i, N}^{n+1}\left[\right.\right.$. Just before disappearing, the moving bottleneck has a velocity equal to $v\left(\rho_{N}^{0}\left(y_{i}^{0}-\right)-n \epsilon_{N}\right)$. The moving bottleneck catches downstream traffic only if its speed is higher that the velocity of downstream traffic. This implies

$$
A t+v\left(\rho_{N}^{0}\left(y_{i}^{0}-\right)\right)>A t_{i, N}^{n}+v\left(\rho_{N}^{0}\left(y_{i}^{0}-\right)\right)=v\left(\rho_{N}^{0}\left(y_{i}^{0}-\right)-n \epsilon_{N}\right)>v\left(\rho_{N}\left(t_{i, N}^{\mathrm{int}}, y_{i, N}\left(t_{i, N}^{\mathrm{int}}\right)+\right)\right) .
$$

The previous inequality yields $\rho_{N}\left(t_{i, N}^{\text {int }}, y_{i, N}\left(t_{i, N}^{\text {int }}\right)+\right)>\rho_{N}^{0}\left(y_{i}^{0}-\right)-n \epsilon_{N}$. Since $\rho_{N}\left(t_{i, N}^{\text {int }}, y_{i, N}\left(t_{i, N}^{\text {int }}\right)+\right) \in \mathcal{M}_{N}$, we have in fact $\rho_{N}\left(t_{i, N}^{\mathrm{int}}, y_{i, N}\left(t_{i, N}^{\mathrm{int}}\right)+\right)-\rho_{N}^{0}\left(y_{i}^{0}-\right) \geq(-n+1) \epsilon_{N}$.

We now need to compare the evolution of the velocity $v\left(\rho_{N}\left(t, y_{i, N}(t)+\right)\right)$ and $A t+v\left(\rho_{N}^{0}\left(y_{i}^{0}-\right)\right)$. Starting from $t_{i, N}^{\text {int }}$, the velocity on the right of the trajectory only increases when it meets a rarefaction wave. We may assume that it will cross a succession of rarefaction waves coming from a given downstream moving bottleneck $j$. When it encounters a first rarefaction wave at time $t$, the density decreases by one grid point, and we then have $\rho_{N}\left(t, y_{i, N}(t)+\right)-\rho_{N}^{0}\left(y_{i}^{0}-\right) \geq-n \epsilon_{N}$. Let us now focus on the second rarefaction encountered, assuming it occurs at time $t^{\prime}>t$. The speed increases of the difference between two consecutive speeds on the mesh, such that $v\left(\rho_{N}\left(t^{\prime}, y_{i, N}\left(t^{\prime}\right)+\right)\right)=v\left(\rho_{N}\left(t, y_{i, N}(t)+\right)-\epsilon_{N}\right)$. In addition, since these rarefactions are generated by a given bottleneck $j$, there exists a time index $k$ such that $v\left(\rho_{N}\left(t, y_{i, N}(t)+\right)-\epsilon_{N}\right)-v\left(\rho_{N}\left(t, y_{i, N}(t)+\right)\right)=$ $A \Delta t_{j, N}^{k}$. In the same time, by construction of the WFT approximations, the second rarefaction was emitted $A \Delta t_{j, N}^{k}$ after the first one, and then the right hand side $A t+v\left(\rho_{N}^{0}\left(y_{i}^{0}-\right)\right)$ will increase by at least $A \Delta t_{j, N}^{k}$ (the second rarefaction travels faster that the first one by concavity of the flux function). This reasoning can then be iterated and combined with (4.3) to ensure that $v\left(\rho_{N}\left(t, y_{i, N}(t)+\right)\right) \leq A t+v\left(\rho_{N}^{0}\left(y_{i}^{0}-\right)\right)$ for any $t>t_{i, N}^{\text {int }}$.

The result of Lemma 6 combined with the definition of $w_{i}(2.2)$ can directly be extended to the limit of the WFT approximations.

Lemma 7. For any $i \in \mathcal{I}$ :

$$
\omega_{i}\left(t, y_{i}(t)\right)=\min \left\{A t+v\left(\rho^{0}\left(y_{i}^{0}-\right)\right), v\left(\rho\left(t, y_{i}(t)+\right)\right\}= \begin{cases}A t+v\left(\rho^{0}\left(y_{i}^{0}-\right)\right), & \text { if } t \leq t_{i}^{i n t} \\ v\left(\rho\left(t, y_{i}(t)+\right)\right), & \text { if } t>t_{i}^{\text {int }}\end{cases}\right.
$$

where $t_{i}^{\text {int }}$ denotes the limit of a subsequence of $\left\{t_{i, N}^{i n t}\right\}_{N \in \mathbb{N}}$. 
Proof. By (2.2) we know that

$$
\omega_{i}\left(t, y_{i}(t)\right)=\min \left\{A t+v_{i}^{0}, v\left(\rho\left(t, y_{i}(t)+\right)\right\} .\right.
$$

On the other hand, for each $N \in \mathbb{N}$, Definition 4 and Lemma 6 directly gives

$$
\dot{y}_{i, N}(t)=\min \left\{A t_{i, N}^{n}+v\left(\rho_{N}^{0}\left(y_{i, N}^{0}-\right)\right), v\left(\rho_{N}\left(t, y_{i, N}(t)+\right)\right\}= \begin{cases}A t_{i, N}^{n}+v\left(\rho_{N}^{0}\left(y_{i, N}^{0}-\right)\right), & \text { if } t \leq t_{i, N}^{\text {int }}, \\ v\left(\rho_{N}\left(t, y_{i, N}(t)+\right)\right), & \text { if } t>t_{i, N}^{\text {int }},\end{cases}\right.
$$

for $t_{i, N}^{n}$ defined in (3.5). Passing to the limit in (4.5), by Lemma 3 and the convergence of speed traces also in the presence of non-classical shocks $[15,26]$, one gets

$$
\min \left\{A t+v\left(\rho^{0}\left(y_{i}^{0}-\right)\right), v\left(\rho\left(t, y_{i}(t)+\right)\right\}= \begin{cases}A t+v\left(\rho^{0}\left(y_{i}^{0}-\right)\right), & \text { if } t \leq t_{i}^{\text {int }} \\ v\left(\rho\left(t, y_{i}(t)+\right)\right), & \text { if } t>t_{i}^{\text {int }}\end{cases}\right.
$$

which provides the required result.

Proposition 1. For any $i \in \mathcal{I}, y_{i}$ is a Carathéodory solution of $(2.1 \mathrm{~d})-(2.1 \mathrm{e})$, i.e.

$$
y_{i}(t)=y_{i}^{0}+\int_{0}^{t} \omega_{i}\left(s, y_{i}(s)\right) \mathrm{d} s \quad \text { for any } t \in[0, T] .
$$

Proof. Let $t_{i}^{\text {int }}$ be the limit of a subsequence of $\left\{t_{i, N}^{\text {int }}\right\}_{N \in \mathbb{N}}$ and fix $\delta>0$ sufficiently small. Assume first that $t \leq t_{i}^{\text {int }}-\delta$. For $N$ large enough, the i-th bottleneck is active on the interval $[0, t]$ and we have $\dot{y}_{i, N}(t)=v\left(\rho_{N}^{0}\left(y_{i}^{0}-\right)-n \epsilon_{N}\right)$ for all $t \in\left[t_{i, N}^{n}, t_{i, N}^{n+1}[\right.$.

We can write

$$
y_{i, N}(t)=y_{i}^{0}+\int_{0}^{t} \dot{y}_{i, N}(s) \mathrm{d} s .
$$

By construction of each approximated parabola $y_{i, N}$ (see [20] for details) we have for $s \in[0, t]$

$$
\lim _{N \rightarrow+\infty} \dot{y}_{i, N}(s)=A s+v\left(\rho^{0}\left(y_{i}^{0}-\right)\right) \leq v\left(\rho\left(y_{i}+\right)\right)=V_{\max } .
$$

We can then apply the dominated convergence theorem to pass to the limit within the integral and combine with Lemma 7 to obtain (4.7).

Now assume that $t>t_{i}^{\text {int }}+\delta$. In this case, the moving constraint is not active anymore, and $\rho_{N}, \rho$ are respectively approximated and weak entropy solutions of (2.1a) on the time interval $\left[t_{i}^{\text {int }}+\delta, t\right]$. The convergence of traces is therefore assured, see e.g. [6, Section 4.3] and [10], and the result (4.7) is straightforward.

4.4. Proof of (2.1c).

Lemma 8. The limit $\rho$ satisfies the flux constraint (2.1c):

$$
f\left(\rho\left(t, y_{i}(t)\right)\right)-\rho\left(t, y_{i}(t)\right) \dot{y}_{i}(t) \leq 0, \quad t>0, \forall i \in \mathcal{I} .
$$

Proof. By construction of the WFT approximations, we have that

$$
f\left(\rho_{N}\left(t, y_{i, N}(t)\right)\right)-\rho_{N}\left(t, y_{i, N}(t)\right) \dot{y}_{i, N}(t) \leq 0, \quad t>0, \forall i \in \mathcal{I}, \forall N \in \mathbb{N} .
$$

We know that for any $N$, the function $\rho_{N}$ is a weak solution of the approximated problem

$$
\begin{cases}\partial_{t} \rho+\partial_{x} f_{N}(\rho)=0, & x \in \mathbb{R}, t>0 \\ \rho(0, x)=\rho_{N}^{0}(x), & x \in \mathbb{R} .\end{cases}
$$

Thus we have $\forall N, \forall \phi \in \mathcal{C}_{c}^{1}([0, T] \times \mathbb{R} ; \mathbb{R})$,

$$
\int_{0}^{T} \int_{-\infty}^{\infty}\left(\rho_{N} \phi_{t}+f\left(\rho_{N}\right) \phi_{x}\right) \mathrm{d} x \mathrm{~d} t+\int_{-\infty}^{\infty} \rho_{N}^{0}(x) \phi(0, x) \mathrm{d} x=0,
$$


since $f_{N}\left(\rho_{N}(t, x)\right)=f\left(\rho_{N}(t, x)\right)$. Now, consider a time-space domain $] 0, T\left[\times \Omega_{i}, \Omega_{i}=\{] y_{i}(t)-a, y_{i}(t)+b[, t \in\right.$ ] $0, T[\}$, where $a, b>0$ are sufficiently small, so that $\Omega_{i}$ contains only the $i$-th moving bottleneck. By uniform convergence, $\Omega_{i}$ contains also all $y_{i, N}$, for $N$ sufficiently large (up to a subsequence). The idea is to change space variables, center the solution around the moving bottleneck, and use the Green-Gauss theorem (see e.g. [2, section 5]). We define

$$
\left.\tilde{\rho}_{i, N}(t, x):=\rho_{N}\left(t, x+y_{i}(t)\right) \quad \text { for } x \in\right]-a, b[, t \in] 0, T[.
$$

Then $\tilde{\rho}_{i, N}$ is a weak solution of

$$
\partial_{t} \tilde{\rho}_{i, N}(t, x)+\partial_{x}\left[f\left(\tilde{\rho}_{i, N}\right)(t, x)-\dot{y}_{i, N}(t) \tilde{\rho}_{i, N}(t, x)\right]=0 .
$$

Let $\psi \in \mathcal{C}_{c}^{1}([0, T])$ a compact test function of time, and $\xi \in \mathcal{C}_{c}^{1}(]-a, b[)$ a compact test function of time such that $\xi(0)=1$. Applying the weak formulation of (4.10) and the Green Gauss theorem, we obtain

$$
\begin{aligned}
\int_{0}^{T} \int_{-a}^{0} \tilde{\rho}_{i, N}(t, x) \dot{\psi}(t) \xi(x) & +\left[f\left(\tilde{\rho}_{i, N}\right)(t, x)-\dot{y}_{i, N}(t) \tilde{\rho}_{i, N}(t, x)\right] \psi(t) \xi^{\prime}(x) \mathrm{d} x \mathrm{~d} t \\
= & \int_{0}^{T}\left(f\left(\tilde{\rho}_{i, N}\right)(t, 0-)-\dot{y}_{i, N}(t) \tilde{\rho}_{i, N}(t, 0-)\right) \psi(t) \mathrm{d} t \leq 0 .
\end{aligned}
$$

Calling $\tilde{\rho}_{i}(t, x)=\lim _{N \rightarrow+\infty} \tilde{\rho}_{i, N}(t, x)$, we can pass to the limit in (4.11) and obtain

$$
\begin{aligned}
0 & \geq \lim _{N \rightarrow+\infty} \int_{0}^{T} \int_{-a}^{0} \tilde{\rho}_{i, N}(t, x) \dot{\psi}(t) \xi(x)+\left[f\left(\tilde{\rho}_{i, N}\right)(t, x)-\dot{y}_{i, N}(t) \tilde{\rho}_{i, N}(t, x)\right] \psi(t) \xi^{\prime}(x) \mathrm{d} x \mathrm{~d} t \\
& =\int_{0}^{T} \int_{-a}^{0} \tilde{\rho}_{i}(t, x) \dot{\psi}(t) \xi(x)+\left[f\left(\tilde{\rho}_{i}\right)(t, x)-\dot{y}_{i}(t) \tilde{\rho}_{i}(t, x)\right] \psi(t) \xi^{\prime}(x) \mathrm{d} x \mathrm{~d} t \\
& =\int_{0}^{T}\left(f\left(\tilde{\rho}_{i}\right)(t, 0-)-\dot{y}_{i}(t) \tilde{\rho}_{i}(t, 0-)\right) \psi(t) \mathrm{d} t .
\end{aligned}
$$

This ensures convergence of the traces along the moving constraint. We can then revert back to the original coordinates in order to obtain (4.8).

5. Numerical examples. To illustrate the model's behavior, we provide in this section numerical simulations of the solutions to the LWR model (1.1) and to our coupled PDE-ODE model (2.1) obtained via the Wave-Front Tracking method. Our numerical code is entirely based on Algorithm 1. Since we mainly expect acceleration and deceleration effects in urban areas, we choose to represent a stretch of road by a segment $[0, L]$ measuring $L=1000 \mathrm{~m}$. This road contains two traffic lights, respectively located at $x_{1}=300 \mathrm{~m}$ and $x_{2}=700 \mathrm{~m}$. The first traffic light is initially red, and changes color every 15 seconds. When the traffic light is red, we enforce numerically a zero-flux constraint at its location. More precisely, at the traffic light positions, the PDE-ODE model (2.1) is completed with the fixed constrain problem introduced in [8], see also [9]:

$$
f\left(\rho\left(t, x_{k}\right)\right) \leq q_{k}(t):=\left\{\begin{array}{ll}
f\left(\rho_{\mathrm{cr}}\right) & \text { if the } k \text {-th traffic light is green, } \\
0 & \text { if the } k \text {-th traffic light is red, }
\end{array} \quad k=1,2 .\right.
$$

The sequence of traffic lights is designed to obtain a "green-wave" with the LWR model: the second traffic light turns green as soon as the first vehicle, traveling at maximum speed, reaches it. It also changes color every 15 seconds.

In the two following numerical illustrations, we consider the speed-density function

$$
v(\rho)=V_{\max }\left(1-\frac{\rho}{\rho_{\max }}\right), \quad \rho \in\left[0, \rho_{\max }\right] .
$$

This leads to a quadratic flow-density fundamental diagram of Greenshield's type, with $\rho_{\text {cr }}=\frac{\rho_{\max }}{2}$. As we consider an urban setting, the maximal speed is set to $V_{\max }=50 \mathrm{~km} / \mathrm{h}$. The maximal density corresponds 
to a bumper-to-bumper situation, so we select $\rho_{\max }=200 \mathrm{veh} / \mathrm{km}$. The acceleration rate is fixed to $A=2 \mathrm{~m} / \mathrm{sec}^{2}$, which is a standard value. Finally, the parameter for our density grid is set to $N=8$.

We observe the following results:

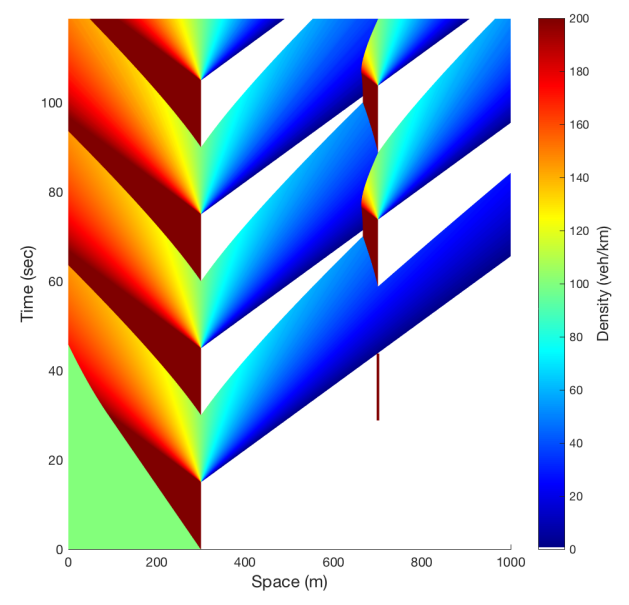

(a) Solution to the LWR model.

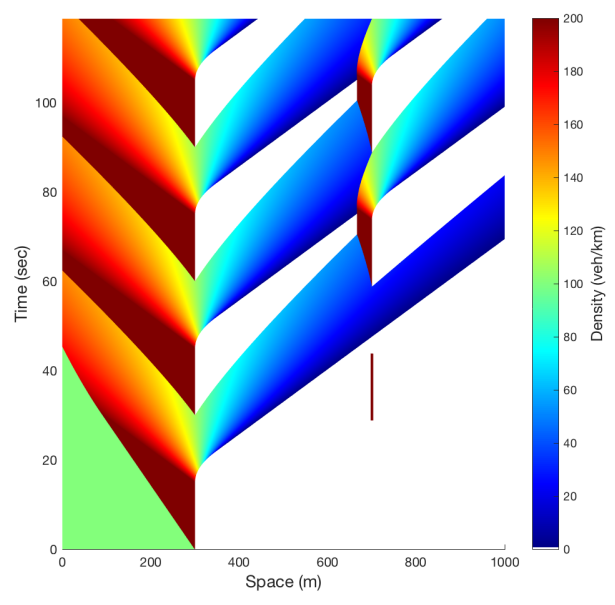

(b) Solution to our PDE-ODE model.

Fig. 5.1: Comparison of solutions for the same sequence of traffic lights.

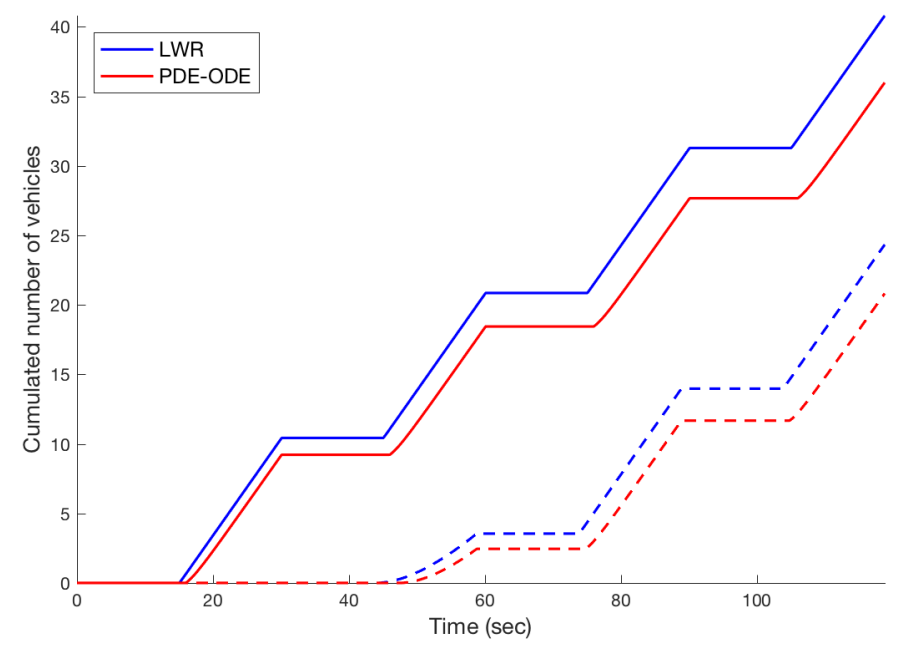

Fig. 5.2: Number of vehicles passing through the first (plain lines) and second (dashed lines) traffic light with respect to time.

As illustrated in Figure 5.2, we observe that the flow moving downstream both traffic lights is significantly more important with the LWR model than with our PDE-ODE model, with differences up to $15 \%$. As a consequence, the queues upstream each traffic light are longer with our model. At this step, since we did not compare models against real traffic data, we cannot assert that (2.1) is more realistic than the classical LWR model. Nonetheless, the simulations reveal that optimizing traffic light sequences based on the LWR 
model may lead to a relevant drop of capacity and an increase of congestion, because this model assumes that vehicles have unbounded acceleration from zero to the maximal speed. This lack of physical realism may have a significant impact in urban areas, in which traffic encounters multiple flux constraints like traffic lights and stop signs, thus passing through multiple acceleration phases.

6. Conclusion. In this paper, we have proposed a strongly coupled PDE-ODE model that allows to capture the finite acceleration of vehicles, which has been shown to have a significant impact, notably in urban applications. We rigorously proved the existence of solutions of this strongly coupled PDE-ODE system for piecewise constant initial data. We also gave a detailed description of how to construct approximate solutions via the Wave-Front Tracking technique. It would be interesting to extend this result to more general initial data, and to check the stability of the corresponding solutions. From the application point of view, the model could be easily extended to road networks [13]. Besides, even though vehicles have lower acceleration abilities than deceleration ones, we may extend the model to also include bounded braking phases, which would impact the representation of the shock waves in the solution. Finally, we still need to confront our model to realistic traffic data in order to assess its performances compared to the classical LWR model.

\section{References.}

[1] L. Ambrosio, N. Fusco, and D. Pallara. Functions of bounded variation and free discontinuity problems, volume 254. Clarendon Press Oxford, 2000.

[2] B. Andreianov, C. Donadello, and M. D. Rosini. A second-order model for vehicular traffics with local point constraints on the flow. Math. Models Methods Appl. Sci., 26(4):751-802, 2016.

[3] A. Aw and M. Rascle. Resurrection of "second order" models of traffic flow. SIAM J. Appl. Math., 60(3):916-938, 2000.

[4] R. Borsche, R. M. Colombo, and M. Garavello. Mixed systems: ODEs - balance laws. J. Differential Equations, 252(3):2311-2338, 2012.

[5] A. Bressan. Hyperbolic systems of conservation laws, volume 20 of Oxford Lecture Series in Mathematics and its Applications. Oxford University Press, Oxford, 2000.

[6] G. Bretti and B. Piccoli. A tracking algorithm for car paths on road networks. SIAM J. Appl. Dyn. Syst., 7(2):510-531, 2008.

[7] C. Chalons, M. L. Delle Monache, and P. Goatin. A conservative scheme for non-classical solutions to a strongly coupled PDE-ODE problem. Interfaces and Free Boundaries, 19(4):553-570, 2018.

[8] R. M. Colombo and P. Goatin. A well posed conservation law with a variable unilateral constraint. $J$. Differential Equations, 234(2):654-675, 2007.

[9] R. M. Colombo, P. Goatin, and M. D. Rosini. On the modelling and management of traffic. ESAIM Math. Model. Numer. Anal., 45(5):853-872, 2011.

[10] R. M. Colombo and A. Marson. A Hölder continuous ode related to traffic flow. Proceedings of the Royal Society of Edinburgh Section A: Mathematics, 133(4):759-772, 2003.

[11] M. L. Delle Monache and P. Goatin. Scalar conservation laws with moving constraints arising in traffic flow modeling: an existence result. J. Differential Equations, 257(11):4015-4029, 2014.

[12] N. S. Dymski, P. Goatin, and M. D. Rosini. Existence of BV solutions for a non-conservative constrained Aw-Rascle-Zhang model for vehicular traffic. J. Math. Anal. Appl., 467(1):45-66, 2018.

[13] N. S. Dymski, P. Goatin, and M. D. Rosini. Modeling moving bottlenecks on road networks. In Hyperbolic Problems: Theory, Numerics, Applications, volume 10 of AIMS on Applied Mathematics, pages 419-426, 2020. Proceedings of the XVII international conference in Penn State, June 2018.

[14] L. C. Evans. Partial differential equations, volume 19 of Graduate Studies in Mathematics. American Mathematical Society, Providence, RI, second edition, 2010.

[15] M. Garavello, P. Goatin, T. Liard, and B. Piccoli. A multiscale model for traffic regulation via autonomous vehicles. J. Differential Equations, 269(7):6088-6124, 2020.

[16] M. Garavello, K. Han, and B. Piccoli. Models for vehicular traffic on networks, volume 9. American Institute of Mathematical Sciences (AIMS), Springfield, MO, 2016.

[17] M. Garavello and B. Piccoli. Traffic flow on networks, volume 1 of AIMS Series on Applied Mathematics. American Institute of Mathematical Sciences (AIMS), Springfield, MO, 2006. Conservation laws models.

[18] H. Holden and N. H. Risebro. Front tracking for hyperbolic conservation laws, volume 152 of Applied Mathematical Sciences. Springer, Heidelberg, second edition, 2015. 
[19] C. Lattanzio, A. Maurizi, and B. Piccoli. Moving bottlenecks in car traffic flow: a PDE-ODE coupled model. SIAM J. Math. Anal., 43(1):50-67, 2011.

[20] N. Laurent-Brouty, G. Costeseque, and P. Goatin. A coupled PDE-ODE model for bounded acceleration in macroscopic traffic flow models. IFAC-PapersOnLine, 51(9):37 - 42, 2018. 15th IFAC Symposium on Control in Transportation Systems CTS 2018.

[21] J. Lebacque. A two phase extension of the LWR Model based on the boundedness of traffic acceleration. In Transportation and Traffic Theory in the 21st Century, pages 697-718. Emerald Group Publishing Limited, 2002.

[22] J.-P. Lebacque. Two-phase bounded-acceleration traffic flow model: analytical solutions and applications. Transportation Research Record: Journal of the Transportation Research Board, (1852):220-230, 2003.

[23] J.-P. Lebacque, J.-B. Lesort, and F. Giorgi. Introducing buses into first-order macroscopic traffic flow models. Transportation Research Record: Journal of the Transportation Research Board, 1644(1):70-79, 1998.

[24] L. Leclercq. Bounded acceleration close to fixed and moving bottlenecks. Transportation Research Part B: Methodological, 41(3):309-319, 2007.

[25] L. Leclercq. A new numerical scheme for bounding acceleration in the LWR model. In 4th IMA International Conference on Mathematics in Transport, 2007.

[26] T. Liard and B. Piccoli. On entropic solutions to conservation laws coupled with moving bottlenecks. working paper or preprint, June 2019.

[27] T. Liard and B. Piccoli. Well-Posedness for Scalar Conservation Laws with Moving Flux Constraints. SIAM J. Appl. Math., 79(2):641-667, 2019.

[28] M. J. Lighthill and G. B. Whitham. On kinematic waves. II. A theory of traffic flow on long crowded roads. Proc. Roy. Soc. London. Ser. A., 229:317-345, 1955.

[29] H. J. Payne. Models of freeway traffic and control. Mathematical models of public systems, 1971.

[30] P. I. Richards. Shock waves on the highway. Operations Res., 4:42-51, 1956.

[31] M. Treiber and A. Kesting. Traffic flow dynamics. Springer-Verlag Berlin Heidelberg, 2013.

[32] S. Villa, P. Goatin, and C. Chalons. Moving bottlenecks for the Aw-Rascle-Zhang traffic flow model. Discrete Contin. Dyn. Syst. Ser. B, 22(10):3921-3952, 2017.

[33] G. B. Whitham. Linear and nonlinear waves, volume 42. John Wiley \& Sons, 1974.

[34] H. M. Zhang. A non-equilibrium traffic model devoid of gas-like behavior. Transportation Research Part B: Methodological, 36(3):275-290, 2002. 